\title{
Atividade física para crianças até 5 anos: Guia de Atividade Física para a População Brasileira
}

\author{
Physical activity for children up to 5 years old: Physical Activity Guidelines for the \\ Brazilian Population
}

\section{AUTORES \\ Larissa Rosa da Silva ${ }^{1}$ (D) \\ Carla Menêses Hardman² (iD \\ Clarice Lucena Martins ${ }^{3}$ iD \\ Paulo Henrique Guerra ${ }^{4}$ (D) \\ Anastácio Neco de Souza Filho ${ }^{3}$ (iD \\ Daniel da Rocha Queiroz ${ }^{2}$ (D) \\ Daniel Umpierre ${ }^{5}$ (D) \\ Rildo de Souza Wanderley Junior ${ }^{2}$ (D) \\ Fabiana Vieira Santos Azevedo Cavalcante ${ }^{6}$ (ID \\ Paula Fabricio Sandreschi ${ }^{7}$ (D) \\ Pedro Curi Hallal ${ }^{8}$ (D) \\ Mauro Virgilio Gomes de Barros 9 (D) \\ 1 Universidade Estadual do Oeste do Paran Departamento de Educacão Física, Marechal \\ Candido Rondon, Paraná, Brasil. \\ 2 Universidade Federal de Pernambuco, \\ Departamento de Educação Física, Recife, \\ Pernambuco, Brasi. \\ 3 Universidade Federal da Paraíba, Departamento \\ de Educação Física, João Pessoa, Paraíba, Brasil. \\ 4 Universidade Federal da Fronteira Sul, Chapecó, Santa Catarina, Brasil. \\ 5 Universidade Federal do Rio Grande do Sul, Departamento de Saúde Coletiva e Centro de Pesquisa Clínica, Hospital de Clínicas de Porto \\ Alegre, Porto Alegre, Rio Grande do Sul, Brasil. \\ 6 Ministério da Saúde, Secretaria de Atenção \\ Primária à Saúde, Departamento de Promoção da \\ Saúde, Brasília, Distrito Federal, Brasil. \\ 7 Ministério da Saúde, Secretaria de Atenção \\ Primária à Saúde, Departamento de Promocão \\ Primaria à Saude, Departamento de Promoção \\ Aa Saude, Coordenaçao Geral de Promoçấ da \\ Atividade Fisica e Açoes Intersetoriais, Brasília, \\ Distrito Federal, Brasil. \\ 8 Universidade Federal de Pelotas, Departamento de Ginástica e Saúde, Pelotas, Rio Grande do Sul, Brasil. 9 Universidade de Pernambuco, Escola Superior de Educação Física, Recife, Pernambuco, Brasil.}

\section{CONTATO}

Larissa Rosa da Silva

larisilva99@yahoo.com.br

Rua Luis Centenaro, n. 533. Marechal Candido Rondon, Paraná, Brasil.

CEP: 85960-000.

DOI

10.12820/rbafs.26e0213

\section{(cc) BY}

Este trabalho está licenciado com uma Licença

Creative Commons - Atribuição 4.0 Internacional.

\begin{abstract}
RESUMO
Este artigo descreve o processo de construção das recomendações de atividade física para crianças de até cinco anos incluídas no Guia de Atividade Física para a População Brasileira. O desenvolvimento destas recomendações tomou por base as diretrizes propostas pela Organização Mundial da Saúde em 2019. Complementarmente, buscou-se suporte teórico nas seguintes estratégias: 1-revisão de escopo, conduzida de modo a atualizar o corpo de evidências acerca dos correlatos de determinantes da atividade física em crianças de até cinco anos; 2-síntese de guias nacionais de atividade física; 3-escuta com pais e professores, a fim de identificar o grau de dificuldade destes em entenderem as recomendações contidas na proposta apresentada pela Organização Mundial de Saúde, além de barreiras e estratégias para maior envolvimento das crianças em atividades físicas; e, 4-consulta pública. Todas as ações foram desenvolvidas no período de maio a dezembro de 2020, por um grupo de trabalho constituído por pesquisadores com experiência na temática e representantes do Ministério da Saúde. Como resultado, foram incluídas no Guia um total de 35 recomendações, sendo 10 relacionadas aos benefícios, quatro à dose, sete aos tipos de atividades, sete às orientações para prática e outras sete relacionadas à redução de comportamento sedentário. Quando pertinente e possível, estas recomendações foram diferenciadas para crianças do nascimento até um ano de vida, de um e dois anos e de três a cinco anos. A mensagem principal é de que qualquer atividade física é melhor do que nenhuma e de que o comportamento sedentário deve ser reduzido.
\end{abstract}

Palavras-chave: Atividade física; Criança; Pré-escolar; Recomendações.

\section{ABSTRACT}

This paper describes the process of establishing physical activity recommendations for children up to five years old included in the Physical Activity Guidelines for the Brazilian Population. The development of these recommendations was based on the guidelines proposed by the World Health Organization in 2019. Theoretical support was gathered by using the following strategies: 1-Scoping review conducted to update the body of knowledge about the correlates and determinants of physical activity in children 0-5 years old; 2-synthesis of the existing national physical activity guidelines; 3-Online interviews with parents and teachers aiming to identify their degree of difficulty in understanding the recommendations contained in the World Health Organization Physical Activity guidelines and to identify barriers and strategies for improving the involvement of children in physical activities; and, 4-Public consultation. All actions were developed in the period from May to December 2020, by a working group composed of researchers with expertise in this field and representatives of the Ministry of Health. As a result, a total of 35 recommendations were included in the Brazilian guidelines, 10 of which were related to benefits, four to the dose, seven to the types of activities, seven to the strategies for improving practice, and seven others related to the reduction of sedentary behavior. When relevant and possible, these recommendations were differentiated for children from birth to one year of age, 1-2 years, and 3-5 years. The main message is that any physical activity is better than none and that sedentary behavior should be reduced.

Keywords: Physical activity, Children, Preschoolers, Guidelines 


\section{Introdução}

A prática de atividade física está diretamente ligada ao crescimento e desenvolvimento saudáveis, de forma que diversos guias internacionais preconizam sua introdução já nos primeiros meses de vida ${ }^{1-4}$. $\mathrm{Na}$ primeira infância, as atividades físicas podem reduzir o risco de desenvolver obesidade ${ }^{5,6}$, melhorar a qualidade do $\operatorname{sono}^{7}$, a coordenação motora ${ }^{5,8}$, as funções cognitivas $^{5,6,8}$, a saúde cardiometabólica ${ }^{5,6}$ e contribuir para o desenvolvimento de habilidades psicológicas e sociais $^{5,9}$.

A primeira infância é um período de rápido desenvolvimento físico, motor e cognitivo, além de se configurar no momento em que os hábitos relacionados ao estilo de vida começam a ser estabelecidos ${ }^{10-12}$. Entretanto, ao contrário do que sugere o senso comum, de que as crianças são "naturalmente ativas", estudo recente realizado com crianças brasileiras indica elevada prevalência de níveis insuficientes da prática de atividades físicas de intensidade moderada a vigorosa ${ }^{13}$.

Baixos níveis de atividade física na primeira infância podem ter consequências negativas tanto em curto, (e.g., desenvolvimento motor e cognitivo inadequados) $)^{5,8}$, quanto em médio e longo prazo (e.g., maior risco de inatividade física nos ciclos de vida subsequentes) ${ }^{11,12}$. Diante disso, estratégias para a orientação de atividades físicas neste momento da vida são importantes para apoiar os pais ou responsáveis, cuidadores e professores. A elaboração de um guia pode ser uma importante estratégia de apoio à promoção da prática de atividade física, por apresentar ao público-alvo, de modo simples e objetivo, uma mensagem de saúde pública construída a partir das evidências científicas disponíveis. Além disso, as recomendações contidas no Guia poderão orientar o monitoramento e avaliação dos níveis de atividade física em crianças até 5 anos e, consequentemente, direcionar pesquisas e intervenções futuras ${ }^{14,15}$.

A Organização Mundial da Saúde $(\mathrm{OMS})^{4}$, em 2019, apresentou as recomendações direcionadas à prática regular de atividades físicas e comportamento sedentário para crianças até quatro anos. No continente americano, recente revisão identificou 10 guias nacionais (Argentina, Canadá, Chile, Colômbia, Costa Rica, Equador, Estados Unidos da América-EUA, Jamaica, Peru e Uruguai) de atividade física chancelados pelos seus respectivos órgãos nacionais de saúde ${ }^{16}$, sendo que poucos apresentaram recomendações específicas para essa faixa etária. No entanto, em virtude da dimensão territorial e os contrastes culturais e socioeconômicos brasileiros, considerou-se importante estabelecer recomendações adequadas à promoção de atividade física dentro da realidade nacional.

Neste sentido, o Ministério da Saúde propôs o desenvolvimento de recomendações considerando diferentes ciclos de vida, inclusive para crianças do nascimento até os cinco anos. Esta iniciativa preenche uma importante lacuna no tocante à orientação sobre a importância da atividade física nas fases iniciais da vida. O Guia de Atividade Física para a População Brasileira foi construído a partir de uma iniciativa do Ministério da Saúde e foi chancelado pela Sociedade Brasileira de Atividade Física e Saúde. Neste artigo, descreve-se o processo de construção das recomendações de atividade física para crianças até cinco anos incluídas no Guia de Atividade Física para a População Brasileira.

\section{Métodos}

Inicialmente, a construção das recomendações brasileiras de atividade física para crianças na primeira infância foi baseada na análise do conteúdo de documento congênere proposto pela OMS em $2019^{4}$. Complementarmente, buscou-se suporte teórico obtido a partir das seguintes estratégias: (1) revisão de escopo, a qual foi conduzida de modo a atualizar o corpo de evidências sobre correlatos e determinantes da atividade física em crianças até 5 anos; (2) síntese de guias nacionais de atividade física de países do continente americano; (3) escuta com pais e professores, a fim de identificar o grau de dificuldade destes em entenderem as recomendações propostas pela OMS, em 2019, além de barreiras específicas para maior envolvimento das crianças em atividades físicas comuns nas diferentes regiões do país; e, (4) consulta pública, a fim de permitir que a sociedade pudesse contribuir com a construção das recomendações mediante apresentação de críticas e sugestões. Na Figura 1, ilustra-se o processo de construção das recomendações, identificando as estratégias e períodos de realização.

As ações foram desenvolvidas por um grupo de trabalho composto por 11 participantes (pesquisadores vinculados a instituições de ensino superior, representantes do Ministério da Saúde e membros do comitê científico do projeto). As atividades foram realizadas no período de maio a dezembro de 2020, abrangendo revisões de literatura, análise de dados, elaboração de relatórios, construção de formulários e reuniões semanais mediante utilização de recursos de videoconfe- 


\section{ESTRATÉGIAS}

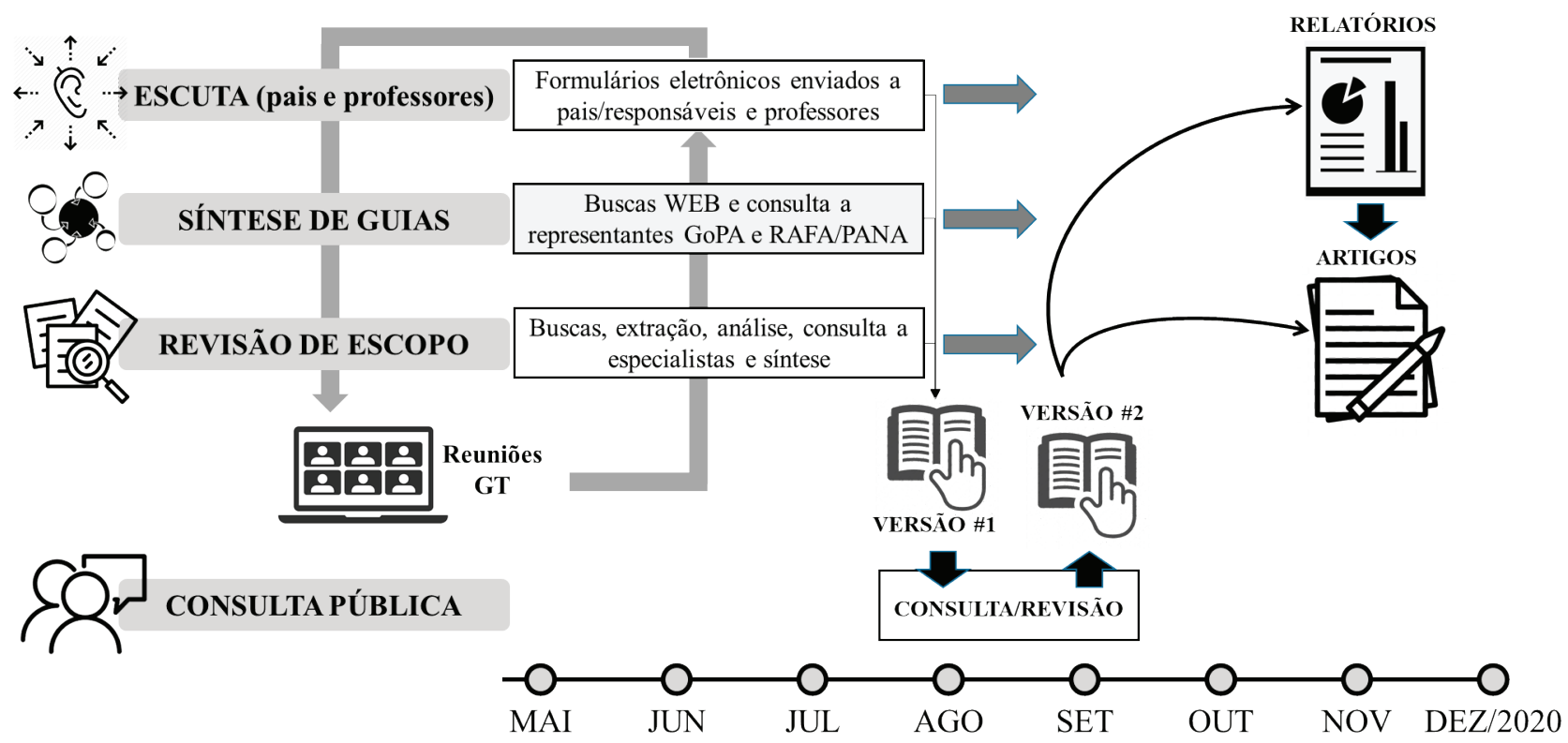

Figura 1 - Resumo das etapas realizadas para a construção das Recomendações brasileiras de atividade física para crianças até 5 anos. GT = Grupo de Trabalho; GoPA: Observatório Global de Atividade Física; RAFA/PANA: Rede de Atividade Física das Américas.

rência. Recomendações específicas para crianças com deficiências e referentes à participação das mesmas nas aulas de educação física escolar foram elaboradas por grupos de trabalhos independentes e estão incluídas no Guia de Atividade Física para a População Brasileira, por isso não foram abordadas neste artigo.

A revisão de escopo objetivou sintetizar os resultados dos estudos sobre correlatos e determinantes da atividade física e do comportamento sedentário realizados com amostras de crianças até 5 anos em países da América do Sul. A preocupação de realizar essa síntese decorreu da identificação de que as recomendações da $\mathrm{OMS}^{4}$ foram baseadas em estudos realizados em outros países, não incluindo os achados derivados de projetos de pesquisa desenvolvidos no Brasil, possivelmente em decorrência das estratégias de busca adotadas e do idioma de publicação. A delimitação da busca a estudos realizados em países da América do Sul foi decorrente da maior similaridade desses contextos em comparação às características observadas no Brasil.

Os procedimentos metodológicos adotados seguiram as recomendações do Preferred Reporting Items for Systematic Review and Meta-Analyses [PRISMA] ${ }^{17}$, recorrendo-se as buscas nas seguintes bases de dados: Literatura Latino-Americana e do Caribe em Ciências da Saúde (Lilacs), PubMed, Scientific Electronic Library Online (Scielo), Scopus e Web of Science. Adicio- nalmente, realizaram-se buscas manuais nas referências bibliográficas das revisões sobre o tema localizadas nas bases de dados e efetuou-se consulta a um painel de 48 pesquisadores que foram identificados como autores de publicações afetas ao tema.

Foi conduzida pesquisa documental a fim de analisar as recomendações de atividade física para crianças na primeira infância apresentadas em guias ou diretrizes nacionais existentes nos 36 países do continente americano. Como critérios de inclusão, procurou-se por guias de atividade física chancelados por agências nacionais de educação, esporte ou saúde, que contivessem recomendações específicas para atividade física direcionadas às crianças na primeira infância. Para tanto, distintas estratégias de busca foram utilizadas: (1) pesquisas manuais nos sítios eletrônicos das agências nacionais de educação, esporte e saúde dos 36 países americanos; (2) pesquisas manuais complementares usando o buscador Google mediante uso dos termos “atividade física”, "primeira infância”, "pré-escolares", "tempo de tela", "comportamento sedentário", além do nome do país (e.g., de acordo com as grafias locais); (3) contato, via e-mail, com os integrantes da Rede de Atividade Física das Américas (RAFA/PANA; https://rafapana.org/), questionando sobre a existência e, nos casos positivos, solicitando documentos locais; e, (4) contato com os responsáveis indicados pelos coun- 
try cards do Observatório Global de Atividade Física (http://www.globalphysicalactivityobservatory.com/ country-cards/), questionando sobre a existência e, nos casos positivos, solicitando documentos locais.

Os documentos localizados foram examinados, procurando-se extrair as recomendações existentes para crianças até 5 anos, além de informações sobre o suporte teórico-científico que apoiou a proposição da mesma. Observou-se, ainda, aspectos relativos à linguagem empregada na apresentação das recomendações e uso de recursos visuais para reforçar mensagens importantes. Mais tarde, na elaboração do relatório final, recorreu-se a uma análise comparativa entre as recomendações existentes nesses guias e aquelas que foram incluídas na versão brasileira.

Estabeleceu-se a priori que o Guia de Atividade Física para a População Brasileira deveria ser apresentado em linguagem informativa e acessível a públicos-alvo da população brasileira como um todo. No caso de crianças até 5 anos, definiu-se que tais recomendações deveriam focalizar os pais ou responsáveis e os professores das crianças. Por essa razão, realizou-se um processo de escuta a voluntários que pudessem representar esses públicos-alvo. A estratégia utilizada buscou garantir a participação desses grupos estratégicos de diferentes regiões do país e com diferentes perfis demográficos e socioeconômicos.

O processo de escuta objetivou identificar, a partir da percepção de pais ou responsáveis e de professores, quais as barreiras e os facilitadores para atividades físicas e comportamentos sedentários em crianças brasileiras do nascimento ao término da primeira infância. Além disso, a escuta também verificou o entendimento destes acerca das recomendações de atividade física e de comportamento sedentário propostas pela $\mathrm{OMS}^{4}$. Em especial, havia interesse de identificar se os participantes conseguiriam entender as recomendações de intensidade e duração das atividades.

Para estruturar o instrumento utilizado na escuta, considerou-se o conhecimento já disponível em revisões sistemáticas existentes sobre o tema ${ }^{5,18,19}$. Após a definição de versão preliminar do instrumento, este foi submetido a uma testagem piloto, na qual um grupo restrito de pais e professores foi convidado a colaborar com o preenchimento do formulário, com a finalidade de verificar a aplicabilidade do mesmo e a compreensão das perguntas. Com base nas informações identificadas no piloto, foram elaborados dois questionários administrados ao respondente via plataforma Google
Forms, sendo um direcionado aos pais, responsáveis ou cuidadores e outro aos professores de crianças até cinco anos. O questionário direcionado aos pais, responsáveis ou cuidadores continha 26 questões e aquele a ser respondido pelos professores continha 24 questões. Essas questões abordaram a aderência das crianças às recomendações de atividade física e de comportamento sedentário, as estratégias adotadas pelos respondentes para ajudar as crianças a realizar atividade física, as barreiras percebidas e as principais atividades realizadas pelas crianças.

Os links para acessar os formulários e responder ao questionário foram divulgados aos grupos estratégicos nas mídias sociais, contando nessa etapa com a colaboração de pesquisadores vinculados a diversas instituições de ensino superior das cinco regiões geográficas do Brasil. Versões dos formulários utilizados estão disponíveis como documento suplementar.

Após a sistematização das informações derivadas da revisão de escopo, da síntese dos guias já existentes e do processo de escuta, uma primeira versão das recomendações foi submetida à consulta pública. Esta versão preliminar foi apresentada à comunidade científica e à sociedade, sendo que qualquer pessoa interessada poderia apresentar críticas ou fazer sugestões e emendas mediante preenchimento de formulário online, o que ficou disponível para preenchimento no período de 14 a 31 de agosto de 2020 . Todas as sugestões recebidas foram analisadas pelo grupo de trabalho e, quando plausíveis e devidamente justificadas do ponto de vista teórico, foram incorporadas ao texto das recomendações.

\section{Resultados}

As recomendações brasileiras de atividade física para crianças até cinco anos foram elaboradas a partir dos resultados obtidos por diferentes estratégias (ver $\mathrm{Fi}^{-}$ gura 1): (1) revisão de escopo; (2) síntese dos guias nacionais de atividade física de países do continente americano; (3) escuta com públicos-alvo; e (4) consulta pública. Desse processo resultou um conjunto de 35 recomendações que estão apresentadas na Tabela 1. Considerando-se que a apresentação de todos os achados resultantes da revisão de escopo e da síntese dos guias nacionais de atividade física de países do continente americano extrapolam o objetivo deste trabalho, tais resultados serão apresentados com maior detalhamento em publicações futuras.

De modo geral, ao analisar os 18 artigos incluídos na revisão de escopo, observou-se que a maioria dos es- 
Tabela 1 - Recomendações de atividade física para crianças até 5 anos e justificativa para inclusão no Guia de Atividade Física para a População Brasileira

\begin{tabular}{ll}
\hline Recomendação & Justificativa \\
\hline Benefícios & \\
\hline
\end{tabular}

A atividade física é importante para todas as crianças, inclusive para os bebês.

O crescimento e o desenvolvimento saudáveis são os principais benefícios da atividade física na infância.

Atividade física auxilia no controle do peso adequado e na diminuição do risco de obesidade.

Melhora a qualidade do sono.

Auxilia na coordenação motora.

Melhora as funções cognitivas e a prontidão para

Ajuda na integração e no desenvolvimento de habilidades psicológicas e sociais.

Contribui para o crescimento saudável de músculo e ossos.

Melhora a saúde do coração e a condição física.

Crianças que são fisicamente ativas tendem a manter a prática de atividade física ao longo da vida. o aprendizado.

Apesar das evidências científicas indicarem os benefícios da AF para as crianças, existe uma suposição (ou crença equivocada) dos pais de que crianças são naturalmente ativas, ou que são muito pequenas para realizar AF, conforme observado na escuta. Adicionalmente, diferentes Guias de AF destacam a importância de incentivar as crianças, inclusive os bebês, a serem ativos, mesmo que por curtos períodos, várias vezes ao dia, em um ambiente seguro, supervisionado e estimulante.

Os primeiros anos de vida são um período crítico e rápido de desenvolvimento físico, cognitivo, social e emocional. Estudos de revisão e algumas diretrizes indicam que o cumprimento das recomendações de AF contribui para o crescimento e o desenvolvimento saudáveis.

Embora estudos e Guias de AF citem os benefícios da AF no controle do peso ou na adiposidade, os achados ainda são controversos relativamente à primeira infância. Enquanto, alguns estudos têm reportado uma relação inversa entre a prática de $\mathrm{AF}$ e os indicadores de adiposidade, outros não observaram essa relação. Apesar da qualidade das evidências ser de muito baixa a baixa, e não ser possível sugerir a dose necessária, os benefícios da AF no controle do peso corporal em longo prazo justificam sua recomendação.

O sono é crucial para a saúde e o desenvolvimento das crianças. Estudo de síntese evidencia que o tempo gasto em atividades físicas moderadas a vigorosas e participação em mais brincadeiras ao ar livre podem melhorar o sono de crianças. No entanto, a qualidade das evidências disponíveis foi muito baixa. Crianças que realizam brincadeiras ao ar livre estão mais expostas à luz natural e isso pode contribuir para regular a secreção de melatonina e o ritmo circadiano, incentivando o início regular do sono e melhorando o sono noturno.

Apesar da qualidade das evidências disponíveis variarem de muito baixa a baixa, outros guias e diferentes estudos também indicam este benefício. Crianças nessa faixa estão em um estágio crucial de aquisição de competência motora. Na primeira infância, a AF parece se associar à melhoria da competência motora e estudos de síntese e guias de AF destacam os benefícios da AF no desenvolvimento motor (habilidades motoras e coordenação motora). O padrão e a força da relação entre esses indicadores tendem a diferir por gênero, indicadores da $\mathrm{AF}$ e medidas de desempenho motor.

Evidências indicam que as atividades físicas contribuem nas funções cognitivas e no aprendizado por facilitar a exploração do ambiente. Estudos de síntese e outros Guias também indicam mudanças positivas no aprendizado da linguagem, no desempenho acadêmico, na atenção e na função executiva com a prática de atividades físicas, entretanto, a qualidade das evidências disponíveis variou de muito baixa a alta

Os estudos disponíveis indicam que as atividades físicas típicas dessa fase da vida (usualmente jogos e brincadeiras) contribuem na saúde psicossocial (autoeficácia, autoestima, temperamento, agressão, integração social). Estas atividades contribuem na formação de estruturas neurais necessárias para que as atividades experimentadas possam ser futuramente repetidas; contribuem para a comunicação e negociação social; e constituem uma possibilidade de expressar emoções. A qualidade das evidências disponíveis variou de baixa a moderada.

As evidências variaram de muito baixa a baixa, no entanto, estudos de síntese e outros Guias de AF indicam que maiores quantidades de AF estão associadas a melhores indicadores de saúde óssea. A infância é um período crítico para o desenvolvimento da estrutura e da massa óssea. A restrição de oportunidades para a prática de atividades físicas pode constituir um importante fator de risco à saúde, com repercussões na saúde física das crianças. Estudos de síntese e outros Guias de atividade física indicam que maiores quantidades de atividade física estão associadas a melhores indicadores de saúde óssea, no entanto, observa-se que a qualidade das evidências disponíveis variou de muito baixa a baixa. Até o momento não se sabe a quantidade de atividade necessária para melhorar as medidas de saúde óssea (ex. densidade e conteúdo mineral ósseo) em crianças com menos de 6 anos de idade.

A qualidade das evidências disponíveis é muito baixa, embora estudos de síntese e outros Guias de AF citem os benefícios da prática de AF na saúde cardiometabólica e na condição física, os achados ainda são limitados e inconsistentes.

A infância representa uma fase crítica durante a qual a conduta das crianças em relação à atividade física é estabelecida, tornando-se mais estáveis e gradualmente mais difíceis de modificar ao longo do tempo. De modo geral, os estudos de acompanhamento dos padrões de atividade física indicam estabilidade dos indicadores da atividade física em crianças. A qualidade das evidências variou de baixa a moderada.

\section{Dose}

Orientação contida em outros Guias (África do Sul, Austrália, Canadá, Reino Unido, OMS). Os bebês devem ser fisicamente ativos várias vezes ao dia, particularmente por meio de brincadeiras interativas no

Até 1 ano: pelo menos 30 minutos por dia de dia enquanto acordados. $\mathrm{Na}$ escuta, observou-se que todos os pais ou responsáveis $(\mathrm{n}=20)$ entenderam a recomendação e a maioria $(n=18)$ relatou que as crianças cumprem essa recomendação. 
Continuação da Tabela 1 - Recomendações de atividade física para crianças até 5 anos e justificativa para inclusão no Guia de Atividade Física para a População Brasileira

\begin{tabular}{ll}
\hline Recomendação & Justificativa \\
\hline Benefícios & \\
\hline $\begin{array}{l}\text { De } 1 \text { a } 2 \text { anos: pelo menos } 3 \text { horas por dia de } \\
\text { atividades físicas de qualquer intensidade, podendo } \\
\text { ser distribuídos ao longo do dia. }\end{array}$ & $\begin{array}{l}\text { Orientação contida em outros Guias (África do Sul, Austrália, Canadá, Reino Unido, OMS). Na escuta, } \\
\text { observou-se que todos os pais ou responsáveis (n=51) entenderam a recomendação e a maioria (n=40) } \\
\text { relatou que as crianças cumprem essa recomendação. }\end{array}$
\end{tabular}

De 3 a 5 anos: pelo menos 3 horas por dia de atividades físicas de qualquer intensidade, sendo, no mínimo, 1 hora de intensidade moderada a vigorosa que pode ser acumulada ao longo do dia.

Quanto mais atividade física, melhor para saúde.

Orientação contida em outros Guias (África do Sul, Austrália, Canadá, Reino Unido, OMS). Na escuta, observou-se que todos os pais ou responsáveis $(\mathrm{n}=123)$ entenderam a recomendação e a maioria $(66,7 \%$, $\mathrm{n}=82$ ) relatou que as crianças cumprem essa recomendação.

Recomendação contida em outros Guias (Austrália, Canadá, EUA, OMS). Sugere-se que quanto maior a frequência e/ou duração na prática de atividades físicas maior os benefícios à saúde física e mental. Também é recomendado que as crianças sejam estimuladas a realizarem uma variedade de atividades físicas de qualquer intensidade, visando os benefícios à saúde.

Tipos de atividades físicas

Jogos e brincadeiras, atividades estruturadas, como a participação em escolinhas de esportes e em aulas de educação física.

Atividades físicas alegres, seguras, supervisionadas pelos pais ou responsáveis e professores e adequadas à idade da criança.

Até 1 ano: brincadeiras e jogos que envolvam atividades que deixem a criança de barriga para baixo (de bruços) ou sentada movimentando braços e pernas e que estimulem a criança a alcançar, segurar, puxar, empurrar, engatinhar, rastejar, rolar, equilibrar-se com ou sem apoio, sentar e levantar, entre outras.

De 1 a 2 anos: brincadeiras e jogos que envolvam atividades como equilibrar nos dois pés, equilibrar num pé só, girar, rastejar, andar, correr, saltitar, escalar, pular, arremessar, lançar, quicar e segurar, entre outras.

De 3 a 5 anos: brincadeiras e jogos que envolvam atividades como caminhar, correr, girar, chutar, arremessar, saltar e atravessar ou escalar objetos, entre outras. Nessa idade, a atividade física também pode ser realizada na aula de educação física escolar, natação, ginástica, lutas, danças e esportes. Ainda, por meio do deslocamento ativo a pé ou de bicicleta, sempre acompanhado dos pais ou responsáveis.

Crianças devem ser fisicamente ativas em vários momentos do dia.

É aconselhável que a criança aprenda a nadar, flutuar, mergulhar e se adaptar ao meio aquático.

Crianças podem ser fisicamente ativas dentro de casa, participando de atividades com familiares, principalmente quando não for possível fazer atividades ao ar livre. acidentes aquáticos, como o afogamento. Essa recomendação também esteve presente em outro Guia de atividade física (Uruguai). Durante a escuta pública, os pais e responsáveis apontaram que atividades na piscina e com água estão entre as mais atrativas para as crianças.

Orientações para prática

Pesquisas demonstraram que crianças que participam de atividades estruturadas e nas aulas de educação física são mais ativas. Além disso, outros Guias de atividade física (EUA, Chile, Uruguai) também apresentam essas recomendações de atividades para essa faixa etária. Durante a escuta pública, identificou-se ainda que a escola/creche oferecer aulas de educação física ou atividades motoras complementares pode ser uma boa estratégia para as crianças serem mais ativas.

Observou-se que a participação dos pais em atividades físicas com os filhos estava inversamente associada ao baixo nível de atividade física em pré-escolares. Além disso, outros Guias de atividade física (EUA, Chile, Uruguai) também apresentam essas recomendações. Durante a escuta pública, identificou-se ainda que tanto os pais como os professores apontam terem receio que as crianças se machuquem durante as atividades físicas, o que poderia ser minimizado quando as atividades são supervisionadas e adequadas a idade da criança.

Estudos apresentam a importância da movimentação nessa faixa etária, indicando que crianças sejam estimuladas a realizarem atividades interativas no chão, assim como manter-se na posição de barriga para baixo quando a criança ainda não engatinha visando os benefícios no desenvolvimento associados à atividade física. Além disso, outros Guias de atividade física (Chile, Uruguai) também apresentam essas recomendações. Durante a escuta pública, identificou-se que as crianças nessa faixa etária preferem atividades de rolar, puxar e pegar brinquedos, engatinhar e brincar no chão.

Pesquisas indicam que as crianças dessa faixa etária sejam estimuladas a realizarem uma variedade de atividades de qualquer intensidade e que desenvolvam habilidades motoras, visando os benefícios associados à atividade física. Além disso, outros Guias de atividade física (Chile, Uruguai) também apresentam essas recomendações. Durante a escuta pública, identificou-se que jogos e brincadeiras que envolvam correr e pular são apontadas como as mais atrativas, tanto pelos pais e responsáveis como pelos professores para essa faixa etária.

Pesquisas recomendam que as crianças dessa faixa etária sejam estimuladas a realizarem uma variedade de atividades de qualquer intensidade e que desenvolvam habilidades motoras, visando os benefícios associados à atividade física. Além disso, outros Guias de atividade física (Chile, Uruguai) também apresentam essas recomendações. Durante a escuta pública, identificou-se que a participação da criança em escolinhas esportivas, assim como, ir e voltar da escola de maneira ativa são boas estratégias para estimular a prática de atividade física. Destaca-se ainda, que os jogos e brincadeiras com bola foram apontados como as atividades mais atrativas para essa faixa etária, tanto pelos pais e responsáveis, como pelos professores.

Estudos demonstraram que quanto maior a quantidade de atividade física realizada maiores serão os benefícios alcançados. Além disso, outros Guias de atividade física (Chile, OMS, Uruguai) também apresentam essas recomendações. Durante a escuta pública, identificou-se que organizar vários períodos do dia para brincar com a criança é uma boa estratégia para aumentar a prática de atividade física da criança.

Estudos ressaltam a importância das atividades físicas ao ar livre. Outros Guias (EUA, Canadá e Chile) indicam a importância do estímulo às tarefas domésticas. Pais e responsáveis indicaram envolver seus filhos em tarefas domésticas como estratégia para o aumento da atividade física. 
Continuação da Tabela 1 - Recomendações de atividade física para crianças até 5 anos e justificativa para inclusão no Guia de Atividade Física para a População Brasileira

\begin{tabular}{ll}
\hline Recomendação & Justificativa \\
\hline Benefícios & \\
\hline $\begin{array}{l}\text { Ir de casa para a escola ou creche caminhando } \\
\text { ou pedalando pode ser uma boa forma de incluir } \\
\text { movimento na vida da criança. }\end{array}$ & $\begin{array}{l}\text { Estudos indicam associação positiva entre deslocamento ativo e nível de atividade física. Na escuta } \\
\text { pública, esta foi uma estratégia indicada pelos pais e responsáveis. }\end{array}$ \\
$\begin{array}{l}\text { O apoio dos pais e responsáveis ou dos cuidadores } \\
\begin{array}{l}\text { é fundamental para que as crianças participem de } \\
\text { jogos, brincadeiras e outros tipos de atividade física } \\
\text { que estimulem a responsabilidade e a autonomia. }\end{array}\end{array}$ & $\begin{array}{l}\text { O aumento dos níveis de atividade física da criança. Esta orientação está presente em diferentes Guias } \\
\text { (EUA, Canadá, Uruguai e Chile). }\end{array}$
\end{tabular}

$\mathrm{Na}$ escuta pública, esta estratégia foi indicada por um número elevado de pais ou responsáveis e professores, no intuito de promover a atividade física de crianças pré-escolares.

hábitos fisicamente ativos.

As crianças maiores de 6 meses devem beber água antes, durante e após a prática de atividade física.

Professores devem incluir o movimento, as brincadeiras regionais ativas e os jogos como parte da rotina das atividades escolares.

Quando os pais e responsáveis participam da atividade física com os filhos, aumentam as chances de que essas crianças sejam fisicamente ativas.

Recomendações incluídas no Guia alimentar para a população brasileira

Orientação contida em outros Guias (EUA e Chile), e sugerida para inclusão durante o processo de escuta pública.

Estudos indicam que a participação dos pais nas atividades se associa positivamente com a participação em atividades físicas e com o nível de atividade física dos filhos. Esta orientação está presente em diferentes Guias (EUA, Canadá e Chile).

Comportamento sedentário

O tempo em celular, computador, tablet,

videogame, televisão deve ser reduzido ao máximo.

Até 1 ano, não é recomendado nenhum tempo em frente a esses dispositivos. Crianças de 1 a 5 anos podem utilizar por até 1 hora por dia.

Quanto menor o tempo de permanência em comportamento sedentário, melhor para saúde.

A substituição do comportamento sedentário por atividades físicas deve ser estimulada.

Quando o comportamento sedentário for inevitável, incentive as crianças a fazerem atividades culturais e educativas como pintura, desenho, jogos de encaixe, quebra-cabeças e contação de histórias com movimento.

A diminuição do tempo gasto com a utilização de celular, computador, tablet, videogame e televisão auxilia no controle do peso adequado, melhora o desenvolvimento motor e cognitivo e ajuda no desenvolvimento de habilidades sociais.

Para um desenvolvimento saudável, quando estiverem acordados, os bebês e as crianças não devem permanecer por mais de 1 hora seguida em carrinhos, cadeirinhas e berços.
O comportamento sedentário em excesso, principalmente relacionado ao uso de telas no tempo livre, está associado à diversos indicadores negativos de desenvolvimento humano nos primeiros anos de vida.

Estas recomendações são amparadas por um corpo de evidências científicas. É importante que pais/ responsáveis possam aplicá-la em suas casas, no sentido de favorecer o desenvolvimento integral de seus filhos.

Reconhecendo os prejuízos ao desenvolvimento que estão associados ao excesso de tempo em comportamento sedentário, medidas restritivas e alternativas mais ativas são importantes.

Respeitando contextos e possibilidades, é importante que crianças possam se envolver em atividades mais ativas, mesmo que estas tenham intensidades mais leves. Complementarmente, a orientação é que pais/ responsáveis também participem dos jogos e brincadeiras juntamente com a criança.

O comportamento sedentário com fins educativos está associado ao desenvolvimento cognitivo das crianças. Dessa forma, recomenda-se que, quando o comportamento sedentário for inevitável, exista uma orientação às atividades culturais e educativas.

O excesso de tempo em telas está associado à diversos indicadores negativos de desenvolvimento humano. Mesmo que o comportamento sedentário seja necessário para a realização de muitas tarefas do dia-a-dia, recomenda-se a introdução de quebras, de modo que crianças não fiquem sentadas por muito tempo ininterruptamente. Também, é importante a orientação para que o uso de telas seja mais cultural e educativo.

Evitar que crianças fiquem sentadas e presas em carrinhos, cadeirinhas e berços por mais de uma hora seguida é preferível, visto sua associação com o desenvolvimento motor. Crianças devem ser estimuladas para a realização de movimentos. tudos foi conduzido adotando delineamentos observacionais e foram realizados em três países (Brasil, Chile e Equador). As amostras tiveram foco mais específico em crianças de três a cinco anos de idade. Os estudos sumarizados mostraram que a maioria das crianças foram classificadas como fisicamente ativas, as quais, por outro lado, encontravam-se demasiadamente expostas a comportamentos sedentários. Os fatores associados à atividade física foram o sexo (meninos são fisicamente mais ativos do que as meninas) e a renda familiar (crianças com maior renda familiar têm mais chances de serem fisicamente ativas). Observou-se, ainda, a partir de estudo de intervenção no contexto escolar, que a orientação dos pais para realizar atividades físicas com os filhos e a mudança do currículo escolar reduziram o tempo de comportamento sedentário das crianças nos finais de semana ${ }^{20}$. No entanto, a análise metodológica desses estudos apontou que tanto nos estudos transversais quanto nas intervenções havia um alto risco de viés para a interpretação dos resultados. 
Ao todo, foram encontrados quatro guias, todos chancelados pelas agências de saúde nacionais. Todos os guias incluídos convergiram em relação aos benefícios, como em relação à importância da atividade física para controle do peso e para o crescimento e desenvolvimento saudáveis. Em relação aos tipos e exemplos de atividade física, houve predominância de orientação quanto à oferta de "atividades físicas alegres, seguras, supervisionadas e adequadas à idade" e a "participação em jogos, brincadeiras e atividades estruturadas". Observou-se a existência de orientações para que as crianças sejam estimuladas a realizarem atividades físicas em vários momentos do dia, assim como em relação à importância do apoio e estímulo dos pais, responsáveis ou dos cuidadores para que as atividades físicas possam ser inseridas na rotina das crianças.

Participaram do processo de escuta com públicos-alvo, 194 pais ou responsáveis de crianças até cinco anos de idade, sendo principalmente mães $(72,2 \%)$, com idade média de 36,1 anos ( $\mathrm{DP}=6,5)$, sendo que 64,9\% referiram ter curso de pós-graduação e 63,4\% tinham crianças com idade de três a cinco anos. Em relação às crianças, a maioria frequentava a escola no turno matutino $(32,5 \%)$ e no sistema de ensino privado $(58,2 \%)$. Em relação aos professores, dos 151 participantes, a maior parte das respostas foi de mulheres $(94,7 \%)$, que atuavam no ensino público $(82,5 \%)$, com idades entre 22 e 68 anos. Grande parte dos professores participantes tinham ensino superior completo $(52,3 \%)$ ou pós-graduação $(42,4 \%)$. Os questionários foram respondidos por pessoas de todas as regiões do país, sendo o maior percentual de respostas oriundo da região Nordeste, tanto para pais e cuidadores (50\%), quanto para professores (80\%).

As recomendações de atividade física foram amplamente compreendidas, tanto pelos pais/responsáveis (100\%) quanto pelos professores (98\%). Em relação à aderência das crianças às recomendações de atividade física, notou-se que na percepção de pais ou responsáveis, $72,2 \%$ das crianças atendem às recomendações dos volumes diários de atividades físicas adequadas à idade. Na percepção dos professores, $84,1 \%$ das crianças atenderiam às recomendações de atividade física. As principais estratégias apontadas pelos pais ou responsáveis para que as crianças pratiquem atividade física são a utilização de parques e locais ao ar livre onde a criança pode se envolver em jogos e brincadeiras e a criação de uma rotina de brincadeiras com os filhos. Por outro lado, estes referem como principais barreiras a ausência de outras crianças nas proximidades do local de residência e o cansaço para participar de brincadeiras.

Em relação ao que foi referido pelos professores, estes apontaram que as principais estratégias para aumentar o envolvimento de crianças em atividades físicas abrangem a realização de brincadeiras ao ar livre, a participação nas atividades com as crianças e a organização de sessões de atividades motoras. $\mathrm{O}$ medo de que as crianças se machuquem é a principal barreira reportadas pelos professores para aumentar a prática de atividades físicas neste subgrupo.

Após submissão das recomendações de atividade física para crianças de até 5 anos ao processo de consulta pública foram apresentadas 35 sugestões válidas, das quais 18 foram incorporadas ao texto e outras 16 foram rejeitadas. As principais razões para não acatar sugestões válidas foram a ausência de suporte teórico para o que foi proposto, apresentação de sugestões que não eram adequadas à faixa etária ou que focalizavam outros comportamentos distintos da prática da atividade física, fugindo ao escopo do Guia.

Ao término do processo, o capítulo do Guia de Atividade Física para População Brasileira referente às crianças de até cinco anos de idade foi elaborado considerando um total de 35 recomendações. $\mathrm{Na}$ tabela 1 estão apresentadas, além das recomendações, um pequeno texto sintetizando o suporte teórico e a justificativa para inclusão de cada recomendação no Guia. As recomendações estão organizadas em quatro dimensões: (1) benefícios para saúde; (2) dose; (3) tipos e exemplos; e, (4) orientações para prática. Adicionalmente, foram incluídas recomendações para redução da exposição a comportamento sedentário neste subgrupo, recomendações que se aglutinam numa quinta dimensão.

\section{Discussão}

As recomendações de atividade física para crianças de até cinco anos foram elaboradas para orientar os cidadãos brasileiros sobre como tornar o dia a dia das crianças mais ativo, respeitando características e os contrastes socioculturais de um país de dimensão continental como o Brasil. Tais recomendações estão apresentadas no capítulo 2 do Guia de Atividade Física para a População Brasileira, uma iniciativa do Ministério da Saúde que coloca, mais uma vez, a promoção da atividade física na agenda de saúde pública nacional.

O Guia como um todo foi idealizado para ser um documento escrito em linguagem acessível a diferentes 
públicos-alvo, podendo contribuir para veiculação de uma mensagem clara de saúde pública no que concerne a importância da atividade física para pessoas de todas as idades, inclusive os bebês. Uma das mensagens principais veiculadas nas recomendações para crianças de até 5 anos é a de que as crianças não são "naturalmente ativas" e de que baixos níveis de atividade física e elevada exposição a comportamentos sedentários podem ser observados mesmo em crianças nessa faixa de idade (primeira infância).

As recomendações apresentadas quanto à dose diária de atividade física convergem em relação ao que está apresentado em outros guias internacionais ${ }^{1,3,4}$, inclusive guias de países das Américas ${ }^{2,21}$. Neste sentido, os achados que fundamentaram a proposição das recomendações brasileiras confirmam que crianças menores de um ano devem acumular pelo menos $30 \mathrm{mi}^{-}$ nutos de atividade na posição de bruços (barriga para baixo), enquanto crianças com idade entre um e cinco anos precisam atingir no mínimo 180 minutos por dia de atividade física de qualquer intensidade. Além disso, dos três aos cinco anos é importante incluir no mínimo 60 minutos de atividade física de intensidade moderada a vigorosa ao longo do dia.

As recomendações apresentadas quanto à necessidade de se adotar restrições ao comportamento sedentário também coincidem com o proposto em outros guias internacionais ${ }^{2,4}$. Desta forma, recomenda-se evitar ao máximo que crianças menores de um ano sejam expostas a utilização de dispositivos eletrônicos ou telas. A partir de um ano, recomenda-se não exceder o período de uma hora por dia. Para um desenvolvimento saudável, recomenda-se, ainda, que bebês e crianças não permaneçam por mais de uma hora contínua em carrinhos, cadeirinhas e berços, quando acordados.

Como mencionado, a prática de atividade física nesta faixa etária proporciona inúmeros benefícios ${ }^{5}$, sendo essencial para um crescimento e desenvolvimento saudáveis, visto que os primeiros anos de vida são um período de rápido desenvolvimento físico, cognitivo, social e emocional ${ }^{4}$. Além disso, a primeira infância representa uma fase importante de estímulo à incorporação de hábitos que podem concorrer para a adoção de um estilo de vida ativo nos demais ciclos de vida. É nesta fase que os comportamentos da criança em relação à atividade física são estabelecidos, tornando-se mais estáveis e gradualmente mais difíceis de modificar ao longo do tempo ${ }^{11,12}$.

Estudos que investigaram os determinantes e cor- relatos sociodemográficos, ambientais e comportamentais da prática de atividade física em diferentes localidades e em diferentes regiões geográficas, identificaram prevalências de baixo nível de atividade física distintas nas cinco regiões do Brasil. Além disso, verificou-se que idade e a escolaridade são os dois principais fatores associados à baixo nível de atividade física ${ }^{22}$. De fato, sabe-se que o nível socioeconômico é um importante determinante do desenvolvimento na fase pré-esco$1 \mathrm{ar}^{23}$, especialmente ao considerar as discrepâncias de renda da população brasileira. Adicionalmente, estudos em crianças até cinco anos identificaram que a escolaridade materna ${ }^{24}$, a renda familiar ${ }^{25}$, e o ambiente social ${ }^{26}$ influenciam diretamente nos níveis de atividade física da criança. Portanto, devido às diferenças demográficas e culturais brasileiras, é necessário que as ações de promoção de atividade física contemplem diferentes abordagens em cada uma das cinco regiões do Brasi ${ }^{22}$.

Importante, ainda, destacar que crianças aprendem brincando, inclusive com outras crianças. Por isso, professores devem incluir o movimento, as brincadeiras regionais ativas e os jogos como parte da rotina das atividades escolares. Estudos mostram que a oferta de aulas de Educação Física ou de atividade física estruturada na escola são fatores associados a maiores níveis de atividade física nas crianças ${ }^{26}$. Paralelamente, a participação e envolvimento dos pais durante a atividade física aumentam as chances de a criança ser fisicamente ativa ${ }^{13,18,19}$. Além disso, estudos demonstraram associação positiva entre nível de atividade física dos pais e nível de atividade física da criança ${ }^{24,27}$. Portanto, é importante que os pais e responsáveis participem em jogos, brincadeiras e outros tipos de atividade física com os filhos, recomendação incluída no Guia.

O baixo nível de atividade física é um dos principais fatores de risco para o desenvolvimento de doenças crônicas $^{28}$. No Brasil, um estudo aponta elevadas prevalências de níveis insuficientes de atividade física em adultos nas capitais brasileiras ${ }^{29}$. Pesquisa com crianças brasileiras em idade pré-escolar identificou níveis insuficientes de atividade física já nos primeiros anos de vida $^{13}$. Neste sentido, a OMS lançou o Plano de Ação Global para a Atividade Física 2018-2030, que visa promover ações para tornar as pessoas mais ativas ao longo da próxima década. Dessa forma, estas recomendações podem e devem ser usadas pelos profissionais e gestores(as) de saúde no planejamento de ações e programas que visem a promoção de atividade física de crianças pré-escolares. 
Entretanto, cabe ponderar que a existência do Guia, por si só, não garante o aumento dos níveis de atividade física da população ${ }^{14}$. É imprescindível que estratégias de comunicação e de disseminação sobre as recomendações, bem como ações apropriadas de promoção da atividade física sejam empregadas para gerar um impacto positivo nos indicadores de atividade física do país ${ }^{20}$. Considerando que dados sobre o nível de atividade física em crianças brasileiras em idade pré-escolar são escassos ${ }^{30}$, essas recomendações poderão dar suporte ao monitoramento e avaliação dos níveis de atividade física de crianças até cinco anos, e consequentemente, dar direcionamentos as pesquisas na área ${ }^{14}$. Além disso, as recomendações brasileiras de atividade física para crianças até 5 anos podem servir de base para o estabelecimento de metas e objetivos para políticas nacionais que visem aumentar a atividade física de crianças em idade pré-escolar ${ }^{15}$. Estudos devem ser conduzidos a fim de identificar os diferentes tipos e doses de atividade física nesta fase, além dos benefícios oriundos do aumento na atividade física e redução do comportamento sedentário.

As recomendações para crianças até cinco anos incluídas no Guia de Atividade Física para a População Brasileira veiculam uma mensagem de saúde pública no tocante à orientação para inclusão da prática regular da atividade física na primeira infância. Reforçam as orientações apresentadas em diretriz da $\mathrm{OMS}^{4}$, contextualizando-as em relação às características demográficas e culturais da população brasileira e, indo além, pela inclusão de recomendações específicas que foram estabelecidas com base no conhecimento produzido em estudos realizados no Brasil e na América do Sul.

As recomendações destacam o entendimento de que a prática de qualquer atividade física é melhor do que nenhuma e de que o comportamento sedentário, dentro das possibilidades, deve ser reduzido ao máximo. Para além dos reconhecidos benefícios da atividade física à saúde, também se reconhece que crianças ativas possuem maiores chances de se tornarem adolescentes e adultos ativos. A importância da ludicidade, dos jogos e brincadeiras como tipos de atividades que podem ser estimulados nessa idade tem destaque no conjunto das recomendações. Assim, como o papel que os pais, responsáveis ou cuidadores podem ter no sentido de estimular as crianças a incluírem atividades físicas em sua rotina diária.

Este estudo apresenta algumas limitações a saber: a qualidade metodológica dos estudos identificados na revisão de escopo, assim como o número reduzido de estudos do tipo intervenção, no entanto, este foi apenas um dos processos que deram base para a construção das recomendações. A maior frequência de respondentes da região Nordeste ao processo de escuta a públicos alvo pode ser entendida como uma limitação, no entanto, todas as regiões tiveram respondentes tanto de pais e cuidadores, quanto de professores. Neste sentido, o período de duas semanas para a consulta pública a especialista pode ser entendido como curto para atingir um maior número de respondentes.

Por fim, as recomendações para crianças até cinco anos podem contribuir para melhorar o monitoramento e a avaliação dos níveis de atividade física nessa faixa etária e, consequentemente, servir ao planejamento de ações de promoção da atividade física. É importante destacar que o conteúdo das recomendações precisa ser amplamente divulgado. Gestores e profissionais de saúde podem planejar a disseminação do conteúdo do Guia e dessas recomendações por diferentes estratégias, inclusive nas escolas, nas Unidades de Saúde e na comunidade como um todo.

\section{Conflito de interesse}

Os autores declaram não haver conflito de interesse.

\section{Financiamento}

Este trabalho descreve as recomendações de atividade física para crianças até 5 anos que estão apresentadas no Guia Brasileiro de Atividade Física, o qual foi financiado pelo Ministério da Saúde do Brasil por meio do Termo de Execução Descentralizada $n^{\circ}$ 56/2019 (projeto: 79224219002/2019; processo: 25000.171034/2019-27).

\section{Contribuição dos autores}

Silva LR liderou a redação do manuscrito, além de ter participado da coleta, análise e interpretação dos dados. Hardman CM, Martins CL, Guerra PH, Souza Filho AN, Queiroz DR, Wanderley Junior RS revisaram criticamente o manuscrito e participaram da coleta, análise e interpretação dos dados. Umpierre D, Cavalcante FVSA, Sandreschi PF e Barros MVG revisaram criticamente o manuscrito e participaram da análise e interpretação dos dados. Hallal PC participou da concepção, redação e revisão geral do manuscrito. Todos os autores participaram em todas as etapas do desenvolvimento das recomendações de atividade física para crianças de 0 a 5 anos, além de terem aprovada a versão final do manuscrito.

\section{Agradecimentos}

Os autores agradecem ao Ministério da Saúde, em especial à 
equipe do Departamento de Promoção da Saúde e à Fundação Delfim Mendes Silveira da Universidade Federal de Pelotas. Aos pais e professores que participaram do processo de escuta pública e aos especialistas e profissionais que contribuíram com a revisão crítica do Guia durante o processo de consulta pública.

\section{Referências}

1. Australian Government. Move and play every day: National physical activity recommendations for children 0-5 years. [Internet]. Department of Health. 2010 [cited 2020 Nov 1]. Available from: https://www1.health.gov.au/internet/main/ publishing.nsf/content/npra-0-5yrs-brochure

2. Canadian Society for Exercise Physiology. Canadian 24hour movement guidelines: an integration of physical activity, sedentary behaviour, and sleep [Internet]. Canadian 24-hour movement guidelines for the years (0-4 yeras). 2016 [cited 2021 Jan 21]. Available from: https://csepguidelines.ca/

3. Draper CE, Tomaz SA, Biersteker L, Cook CJ, Couper J, de Milander M, et al. The South African 24-hour movement guidelines for birth to 5 years: An integration of physical activity, sitting behavior, screen time, and sleep. J Phys Act Heal. 2020;17(1):109-20.

4. World Health Organization. Guidelines on physical activity, sedentary behaviour and sleep for children under 5 years of age [Internet]. World Health Organization. Geneva; 2019 [cited 2021 Jan 22]. Available from: https://apps.who.int/ iris/handle/10665/311664

5. Carson V, Lee EY, Hewitt L, Jennings C, Hunter S, Kuzik N, et al. Systematic review of the relationships between physical activity and health indicators in the early years (0-4 years). BMC Public Health. 2017;17(19):33-63.

6. Pate RR, Hillman CH, Janz KF, Katzmarzyk PT, Powell $\mathrm{KE}$, Torres A, et al. Physical activity and health in children younger than 6 years. Med Sci Sport Exerc [Internet]. 2019 Jun 1 [cited 2021 Jan 22]; 51(6):1282-91. Available from: http://journals.lww.com/00005768-201906000-00023

7. Janssen X, Martin A, Hughes AR, Hill CM, Kotronoulas G, Hesketh KR. Associations of screen time, sedentary time and physical activity with sleep in under 5s: A systematic review and meta-analysis. Sleep Med Rev. 2020;49(101226):1-18.

8. Zeng N, Ayyub M, Sun H, Wen X, Xiang P, Gao Z. Effects of physical activity on motor skills and cognitive development in early childhood: A systematic review. Biomed Res Int [Internet]. 2017 [cited 2021 Jan 22]; 2017:1-14. Available from: https://pubmed.ncbi.nlm.nih.gov/29387718/

9. Eaton WO, McKeen NA, Campbell DW. The waxing and waning of movement: implications for psychological development. Dev Rev. 2001;21(2):205-23.

10. Barros SSH, Nahas MV, Hardman CM, Bezerra J, Barros MVG de. Longitudinal follow-up of physical activity from preschool to school age: The ELOS-Pré study. Rev Bras Cineantropometria e Desempenho Hum. 2019;21:1-11.

11. Caldwell HAT, Proudfoot NA, King-Dowling S, Di Cristofaro NA, Cairney J, Timmons BW. Tracking of physical activity and fitness during the early years. Appl Physiol Nutr Metab . 2016;41(5):504-10.

12. Telama R, Yang X, Leskinen E, Kankaanpää A, Hirvensalo $\mathrm{M}$, Tammelin T, et al. Tracking of physical activity from early childhood through youth into adulthood. Med Sci Sports Exerc. 2014;46(5):955-62.
13. Martins CML, Lemos LFGBP, Souza Filho AN, Bezerra TA, Soares IAA, Mota JG, et al. Adherence to 24-hour movement guidelines in low-income Brazilian preschoolers and associations with demographic correlates. Am J Hum Biol [Internet]. 2020 Oct 19 [cited 2021 Jan 15]; e23519. Available from: https://onlinelibrary.wiley.com/doi/10.1002/ ajhb.23519

14. Milton K, Bauman AE, Faulkner G, Hastings G, Bellew $\mathrm{W}$, Williamson $\mathrm{C}$, et al. Maximising the impact of global and national physical activity guidelines: the critical role of communication strategies. Br J Sports Med [Internet]. 2020 Dec 1;54(24):1463 LP - 1467. Available from: http://bjsm. bmj.com/content/54/24/1463.abstract

15. Troiano RP, Stamatakis E, Bull FC, Factor R. How can global physical activity surveillance adapt to evolving physical activity guidelines? Needs, challenges and future directions Risk Factor Assessment. Br J Sport Med [Internet]. 2020 [cited 2021 Jan 11];54(1):1468-73. Available from: http:// bjsm.bmj.com/

16. Guerra PH, Garcia LMT, Ribeiro EHC, Maretti ALR, Andrade DR., Florindo AA. Identificação e análise das diretrizes nacionais de atividade física nas Américas. Cienc Saude Coletiva [periodico online] [Internet]. 2020 [cited 2021 Jan 22];Jun 2020. Available from: http://www. cienciaesaudecoletiva.com.br/artigos/identificacao-eanalise-das-diretrizes-nacionais-de-atividade-fisica-nasamericas $/ 17643$

17. Liberati A, Altman DG, Tetzlaff J, Mulrow C, Gøtzsche PC, Ioannidis JPA, et al. The PRISMA statement for reporting systematic reviews and meta-analyses of studies that evaluate health care interventions: Explanation and elaboration. Vol. 6, PLoS Medicine. 2009.

18. Hesketh KR, Lakshman R, Van Sluijs EMF. Barriers and facilitators to young children's physical activity and sedentary behaviour: a systematic review and synthesis of qualitative literature. Vol. 18, Obesity Reviews. 2017. p. 987-1017.

19. Hoyos-Quintero AM, García-Perdomo HA. Factors related to physical activity in early childhood: A systematic review. J Phys Act Heal. 2019;16(10):925-36.

20. Romo ML, Abril-Ulloa V. Improving nutrition habits and reducing sedentary time among preschool-aged children in Cuenca, Ecuador: A trial of a school-based intervention. Prev Chronic Dis. 2018;15(96):1-9.

21. Gobierno de Chile. Recomendaciones para la práctica de actividad física según curso de vida. Santiago de Chile; 2017.

22. Mielke GI, Malta DC, de Sá GBAR, Reis RS, Hallal PC. Diferenças regionais e fatores associados à prática de atividade física no lazer no Brasil: Resultados da pesquisa nacional de saúde-2013. Rev Bras Epidemiol [Internet]. 2015 Dec 1 [cited 2021 Jan 15];18:158-69. Available from: http://www. scielo.br/scielo.php?script=sci_arttext\&pid=S1415-790X201 5000600158\&lng=en\&nrm=iso\&tlng=pt

23. Lu C, Black MM, Richter LM. Risk of poor development in young children in low-income and middle-income countries: an estimation and analysis at the global, regional, and country level. Lancet Glob Heal [Internet]. 2016 Dec 1 [cited 2021 Jan 15];4(12):e916-22. Available from: www.thelancet.com/ lancetgh

24. Ricardo LIC, Silva ICM da, Leão OADA, Domingues MR, Wehrmeister FC. Objectively measured physical activity in one-year-old children from a Brazilian cohort: Levels, patterns and determinants. Int J Behav Nutr Phys Act. 2019;16(131):1-13. 
25. Goncalves WSF, Byrne R, Viana MT, Trost SG. Parental influences on screen time and weight status among preschool children from Brazil: A cross-sectional study. Int J Behav Nutr Phys Act. 2019;16(27):1-8.

26. Melo EN, Barros M, Reis RS, Hino AAF, Santos CM, Farias Junior JC. O ambiente no entorno da escola está associado ao deslocamento ativo para escola em pré-escolares? Rev Bras Cineantropometria e Desempenho Hum [Internet]. 2013 [cited 2021 Jan 21];15(4):393-404. Available from: http:// dx.doi.org/10.5007/1980-0037.2013v15n4p393

27. Wanderley Júnior R, Hardman C, Oliveira E, Brito A, Barros $\mathrm{S}$, Barros M. Fatores parentais associados à atividade física em pré-escolares: a importância da participação dos pais em atividades físicas realizadas pelos filhos. Rev Bras Ativ Fís Saúde. 2013;18(2):205-14.

28. Hallal PC, Andersen LB, Bull FC, Guthold R, Haskell W, Ekelund U. Global physical activity levels: surveillance progress, pitfalls, and prospects. Lancet [Internet]. 2012;380(9838):247-57. Available from: http://www. sciencedirect.com/science/article/pii/S0140673612606461
29. Streb AR, Matias TS, dos Santos Leonel L, Tozetto WR, Vieira CG, del Duca GF. Association between physical inactivity in leisure, work, commuting, and household domains and nutritional status in adults in the capital cities of Brazil. Rev Nutr [Internet]. 2019 [cited 2021 Apr 2];32. Available from: http://dx.doi.org/10.1590/1678-9865201932e180276

30. Guerra PH, Barbosa Filho VC, Almeida A, Silva L de S, Pinto MTV, Leonel RM, et al. Revisão sistemática dos indicadores de atividade física e de comportamento sedentário em préescolares sul-americanos. Rev Paul Pediatr [Internet]. 2020 [cited 2021 Jan 21];38:1-10. Available from: http://dx.doi. org/10.1590/1984-0462/2020/38/2018112

Recebido: 31/01/2021

Aprovado: $13 / 04 / 2021$

\section{Como citar este artigo:}

Silva LR, Hardman CM, Martins CL, Guerra PH, Souza Filho AN, Oueiroz DR, Umpierre D, Wanderley Junior RS, Cavalcante FVSA, Sandreschi PF, Hallal PC, Barros MVG. Atividade física para crianças até 5 anos: Guia de Atividade Física para a População Brasileira. Rev Bras Ativ Fis Saúde. 2021;26:e0213 DOI: 10.12820/rbafs.26e0213 